\title{
Closed-Loop Slow-Wave tACS Improves Sleep-Dependent Long-Term Memory Generalization by Modulating Endogenous Oscillations
}

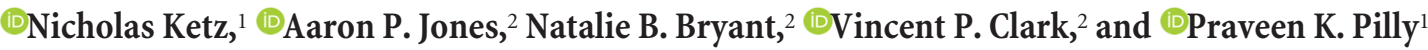 \\ ${ }^{1}$ Information and Systems Sciences Laboratory, Center for Human Machine Collaboration, HRL Laboratories, Malibu, California 90265 and ${ }^{2}$ Psychology \\ Clinical Neuroscience Center, Department of Psychology, University of New Mexico, Albuquerque, New Mexico 87131
}

Benefits in long-term memory retention and generalization have been shown to be related to sleep-dependent processes, which correlate with neural oscillations as measured by changes in electric potential. The specificity and causal role of these oscillations, however, are still poorly understood. Here, we investigated the potential for augmenting endogenous slow-wave (SW) oscillations in humans with closedloop transcranial alternating current stimulation (tACS) with an aim toward enhancing the consolidation of recent experiences into long-term memory. Sixteen (three female) participants were trained presleep on a target detection task identifying targets hidden in complex visual scenes. During post-training sleep, closed-loop SW detection and stimulation were used to deliver tACS matching the phase and frequency of the dominant oscillation in the range of $0.5-1.2 \mathrm{~Hz}$. Changes in performance were assessed the following day using test images that were identical to the training ("repeated"), and images generated from training scenes but with novel viewpoints ("generalized"). Results showed that active SW tACS during sleep enhanced the postsleep versus presleep target detection accuracy for the generalized images compared with sham nights, while no significant change was found for repeated images. Using a frequencyagnostic clustering approach sensitive to stimulation-induced spectral power changes in scalp EEG, this behavioral enhancement significantly correlated with both a poststimulation increase and a subsequent decrease in measured spectral power within the SW band, which in turn showed increased coupling with spindle amplitude. These results suggest that augmenting endogenous SW oscillations can enhance consolidation by specifically improving generalization over recognition or cued recall.

Key words: consolidation; EEG; long-term memory; sleep; tACS

Significance Statement

This human study demonstrates the use of a closed-loop noninvasive brain stimulation method to enhance endogenous neural oscillations during sleep with the effect of improving consolidation of recent experiences into long-term memory. Here we show that transient slow oscillatory transcranial alternating current stimulation (tACS) triggered by endogenous slow oscillations and matching their frequency and phase can increase slow-wave power and coupling with spindles. Further, this increase correlates with overnight improvements in generalization of recent training to facilitate performance in a target detection task. We also provide novel evidence for a tACS-induced refractory period following the tACS-induced increase. Here slow-wave power is temporarily reduced relative to sham stimulation, which nonetheless maintains a positive relationship with behavioral improvements.

\section{Introduction}

Large strides have been made in research surrounding the role of sleep in stabilizing memories. The current model suggests that

Received Jan. 30, 2018; revised May 31, 2018; accepted June 13, 2018.

Author contributions: A.P.J., V.P.C., and P.K.P. designed research; N.K., A.P.J., N.B.B., V.P.C., and P.K.P. performed research; N.K., A.P.J., N.B.B., and P.K.P. analyzed data; N.K., A.P.J., N.B.B., V.P.C., and P.K.P. wrote the paper.

This material is based upon work supported by the DARPA (Defense Advanced Research Projects Agency) and the Army Research Office under Contract No. W911NF-16-C-0018. Any opinions, findings, and conclusions or recommendations expressed in this material are those of the authors and do not necessarily reflect the views of the DARPA or the Army Research Office. recent experiences are reactivated during slow-wave sleep, and this reactivation allows for the integration of these experiences

All authors listed as affiliated with HRL Laboratories were paid employees during the time of research. P.K.P. has a pending patent application on the closed-loop tACS method. The authors declare no other competing financial interests.

Correspondence should be addressed to either Nicholas Ketz or Praveen K. Pilly, Information and Systems Sciences Laboratory, Center for Human Machine Collaboration, HRL Laboratories, Malibu, CA 90265, E-mail: naketz@hrl.com or nick.ketz@gmail.com or pkpilly@hrl.com.

DOI:10.1523/JNEUROSCI.0273-18.2018

Copyright $\odot 2018$ the authors $\quad 0270-6474 / 18 / 387314-13 \$ 15.00 / 0$ 
into long-term memory. The proposed mechanisms supporting this consolidation suggest that during slow-wave sleep the neocortex begins synchronously oscillating in the slow-wave (0.5-1 $\mathrm{Hz})$ and delta bands $(1-4 \mathrm{~Hz})$. These oscillations are made up of periods of neuronal depolarization accompanied by sustained firing ("up states") and periods of hyperpolarization associated with neuronal silence ("down states"). These depolarizing up states allow for thalamocortical spindles $(10-16 \mathrm{~Hz})$ to emerge in the neocortex, which in turn have been shown to synchronize with hippocampal sharp-wave ripples. This has been suggested to facilitate the consolidation of recent experiences, such that ripples indicate hippocampal reactivation, and ripple spindle-slowwave events mark the transfer of that content to the neocortex (Rasch and Born, 2013; Staresina et al., 2015). The causal role of slow-wave (SW) oscillations on spindles and ripples, and the implications of manipulating the endogenous interdependence of each in memory consolidation have yet to be fully explored.

Many studies of sleep-dependent changes in declarative memory show correlations between spindle power and increased "gist" in the learned material rather than recall or recognition. This gist has been captured in various ways, including insight into the hidden structure of digit strings (Wagner et al., 2004), explicit knowledge of hidden serial reaction time task response patterns (Fischer et al., 2006), and integration of new words into existing knowledge (Tamminen et al., 2010). These behavioral changes have been shown to correlate with neural oscillations during sleep, suggesting that altering sleep-related neural oscillations could in turn alter the consolidation of these various experiences into existing knowledge.

Indeed, transcranial alternating current stimulation (tACS) has been shown to enhance sleep-dependent consolidation processes. The dominant paradigms thus far generally use open-loop blocks ( 5 min on, 1 min off) of $0.75 \mathrm{~Hz}$ tACS applied to prefrontal cortex in non-rapid eye movement (REM) sleep stages $\mathrm{N} 2$ and N3. In general, they have shown a selective enhancement for postsleep declarative memory tasks as opposed to procedural memory tasks (Marshall et al., 2006; Westerberg et al., 2015; Ladenbauer et al., 2017). These results, however, have not been without contention. Some transcranial stimulation studies have yielded null results in both behavior and electrocorticography, casting doubts on the capability for tACS to influence neuronal processing without matching endogenous activity (Horvath et al., 2015; Lafon et al., 2017). These results suggest that stimulation-induced changes in sleep processes can impact behavior; however, the specificity of those behavioral changes and the sufficient physiological manipulation are not yet well understood.

Similarly, transcranial direct current stimulation (tDCS) has been shown to influence behavioral performance in a variety of tasks. In particular, Clark et al. (2012) found a stable dose-dependent effect of tDCS on performance such that increasing levels of stimulation improved participants' ability to find hidden targets in complex scenes. This effect was found to persist for at least $24 \mathrm{~h}$; however, no overnight changes in performance were found (Clark et al., 2012; Coffman et al., 2012; Falcone et al., 2012). This raises an intriguing question: if tDCS-enhanced performance was paired with tACS-enhanced sleep-dependent consolidation, could behavioral improvements be further augmented?

This study addresses these open questions by using a novel closed-loop tACS stimulation protocol to target the endogenous SW oscillations during sleep. The influence on behavior is measured in declarative memory through a tDCS-enhanced target detection paradigm where participants must learn subtle cues to detect targets hidden in a complex visual environment. Critically, this paradigm has previously shown no overnight changes in performance and can assess performance in recognition memory as well as generalization. Here we aimed to show that closed-loop tACS would enhance endogenous SW oscillations driving an improvement in overnight memory performance beyond any tDCS-related improvements.

\section{Materials and Methods}

The description of the experimental paradigm and procedures below are also presented in the study by Jones et al. (2018).

\section{Inclusion/exclusion criteria}

Participants were 18-40 years of age, used English as a first language, completed high school, and had no history of head injury with loss of consciousness for $>5 \mathrm{~min}$. They were right handed according to the Edinburgh Handedness Inventory (Oldfield, 1971), had no history of neurological or psychiatric disorder, had no history of alcohol or drug abuse, were nonsmoking, had no excessive alcohol or caffeine consumption, were not currently taking any medication significantly affecting the CNS, had no implanted metal, had no sensitivity or allergy to latex, had good or corrected hearing and vision, and reported no sleep disturbances. Women who were pregnant, or thought they may be, were also excluded.

\section{Participants}

A total of 21 participants, who were recruited using flyers placed around campus of the University of New Mexico and surrounding community, completed the experiment and received monetary compensation. Four subjects were excluded from the analyses due to equipment failure resulting in relatively fewer stimulation events through their active nights $(>1$ SD of stimulation counts across the pool), and one subject was excluded due to an unexpected long gap between the acclimation night and the first experimental night. All participants provided signed informed consent to participate in the study, which was approved by the Chesapeake Institutional Review Board. The remaining $N=16$ participants comprised 3 females, with a mean age of 22.25 years and an SD of 4.96 years.

\section{Target detection paradigm}

A modified version of the original task, described in the study by Clark et al. (2012), which trained subjects to discover the presence of hidden targets in static images and tracked changes in performance through time, was created to allow for the within-subjects design of the current study. A total of 1320 still images were extracted from the videos and edited to include or remove specific objects. Targets that were hidden in these images included explosive devices concealed by or disguised as dead animals (e.g., camels), roadside trash, fruit, flora, rocks, sand, or building structures; and enemies in the form of snipers, suicide bombers, tank drivers, or stone throwers. The stimulus set was divided into the following two target categories: people targets (e.g., enemy snipers, friendly fire) and object targets (e.g., improvised explosive devices, trip wires). Half of the images presented to participants during testing following training were identical to those seen in training (repeated images), and half were related, but with varying spatial perspective from the same corresponding scenes (generalized images). This design allowed for the investigation of effects of the sleep intervention on performance of veridical recall or selective item consolidation in the repeated images, and of multi-item generalization performance in the generalized images (Stickgold and Walker, 2013). Participants were instructed that they could stop the task at any time if the stimuli were too uncomfortable or made them anxious. No subjects elected to stop for such a reason.

\section{Waking electroencephalographic data collection}

Subjects were prepped and fitted with a neoprene electroencephalographic (EEG) cap that incorporated $32 \mathrm{Ag}-\mathrm{AgCl}$ EEG electrodes, placed according to the 10-20 international EEG system (P7, T7, CP5, FC5, F7, F3, C3, P3, FC1, CP1, Pz, PO4, 02, Oz, O1, PO3, CP2, Cz, FC2, Fz, AF3, Fp1, Fp2, AF4, P8, T8, CP6, FC6, F8, F4, C4, and P4). Three external 
channels were used, including electrocardiogram (PO3) placed under the left collarbone, and both vertical (AF3) and horizontal (AF4) electrooculogram: one channel was placed superior and lateral to the right outer canthus, and another was placed inferior and lateral to the left outer canthus. CMS (common mode sense) and DRL (driven right leg) reference electrodes were placed on the preauricular. Data were sampled at $500 \mathrm{~Hz}$.

\section{Sleep polysomnographic data collection}

For polysomnographic (PSG) data collection during sleep, the setup was nearly identical to wake, with a few exceptions. First, two EMG electrodes were placed on and under the chin in accordance with PSG recording guidelines set forth by the American Academy for Sleep Medicine (Berry et al., 2015) to help with sleep scoring. Second, data were collected from 25 EEG electrodes, of which $\mathrm{C} 3, \mathrm{C} 4, \mathrm{O} 1$, and $\mathrm{O}_{2}$ were used for sleep staging. For overnight closed-loop SW sleep augmentation, four channels dedicated for stimulation were used; namely, F3/F4 place in normal $10-20$ positions, and T7/T8 placed on bilateral mastoids. Finally, as F3 and F4 were used for stimulation, they were omitted from data collection; thus, Fp1 and Fp2 were used to assign sleep stages when needed.

\section{Waking transcranial direct current stimulation}

Thirty minutes of continuous tDCS was delivered via the StarStim R32 simultaneous EEG/Stimulation device (Neuroelectrics) during $48 \mathrm{~min}$ of training. A custom tDCS template for use during awake training was defined in the Neuroelectrics control software CoreGUI. In the active condition, a total dose of $1000 \mu \mathrm{A}(1.0 \mathrm{~mA})$ was specified, and for the sham condition a total dose of $100 \mu \mathrm{A}(0.1 \mathrm{~mA})$ was specified. Two electrodes with saline-soaked sponges $\left(25 \mathrm{~cm}^{2}\right)$ were affixed to the participants using a Coban adhesive bandage. In the active condition, the anode electrode was centered over the right sphenoid bone (electrode site F10), and the cathode electrode was placed on the upper contralateral $\mathrm{arm}$. These locations and stimulating amplitudes are based on prior studies using this target detection paradigm (Clark et al., 2012). In the sham condition, the placement, polarities, and duration were identical to the active placement, but the current was set to $0.1 \mathrm{~mA}$ instead of $1.0 \mathrm{~mA}$.

Physical sensation ratings were solicited three times during tDCS administration: once after current ramp-up ( $\sim 1 \mathrm{~min}), 4 \mathrm{~min}$ following ramp-up before the first training run began $(\sim 5 \mathrm{~min}$ after stimulation had begun), and immediately following the first training run $(\sim 21 \mathrm{~min}$ after stimulation had begun). Participants were asked to rate three different types of sensations (itching, heat/burning, and tingling) on a $0-10$ Likert scale, where 0 indicated no feeling of sensation at all and 10 indicated the worst possible feeling of sensation. Any report of a score of $\geq 7$ resulted in immediate cessation of stimulation and termination of the experiment, without penalty to the participant.

\section{Closed-loop transcranial alternating current stimulation during slow-wave sleep}

Illustrated in Figure 2, our closed-loop algorithm for electrical augmentation of slow-wave sleep first detects the presence of SW oscillations, which consist of slow synchronized upward and downward deflections of EEG that are associated with memory consolidation. The algorithm next attempts to match the stimulation frequency and phase with ongoing SW activity such that maximal stimulation occurs at the up states (positive half waves) of the endogenous SW oscillations, as prior work suggests that these are the periods during which coordinated memory replays between hippocampus and neocortex occur to facilitate long-term memory consolidation (Ji and Wilson, 2007). For robust SW detection, a virtual channel is computed by averaging 13 frontocentral EEG channels (Cz, FC1, FC2, CP1, CP2, Fz, C4, Pz, C3, F3, F4, P3, and P4 in the 10-20 system) to determine the overall synchronous activity of EEG recorded during sleep. The virtual channel allows the observation of moments of relatively high SW power, referred to as "SW events," while averaging out the activity of lesser magnitudes on individual channels unrelated to the pattern of SW oscillations. The included channels are stored in a running $5 \mathrm{~s}$ buffer. They undergo moving average subtraction with a $1 \mathrm{~s}$ window (to mean center the signals at $0 \mu \mathrm{V}$ ), and noisy channels exceeding a 500 $\mu \mathrm{V}$ minimum-to-maximum amplitude across the $5 \mathrm{~s}$ are rejected before the virtual channel is computed. The buffer is updated with each discrete data fetch operation that gets the latest data up to the point of that data request. By the time the buffer is updated, there is a random transmission delay, which needs to be accounted for to plan and precisely time the brain stimulation intervention in the near future.

The virtual channel data in the buffer is further processed to actually detect the presence of SW oscillations and possibly predict the upcoming up state. The algorithm applies a fast Fourier transform to these stored data to determine the power spectrum. Stimulation is planned when the ratio of the cumulative power in the SW band $(0.5-1.2 \mathrm{~Hz})$ is $>20 \%$ of the total cumulative power from 0.1 to $250 \mathrm{~Hz}$. If this SW relative power threshold of 0.2 (or $20 \%$ ) is crossed, the algorithm then filters the data in the SW band with a second-order, phase-corrected Butterworth filter. Next, a sine wave is fit to the filtered virtual channel using the identified dominant frequency in the SW band, and with the amplitude, offset, and phase parameter values optimized. The sine wave is then projected into the future, identifying the temporal targets that would synchronize brain stimulation to the predicted endogenous signal. Throughout this process, the dynamic latency associated with data processing is timed using the system clock. Together with distributions of calibrated latencies for data fetch and stimulation commands (mean $=5 \mathrm{~ms}, \mathrm{SD}=2 \mathrm{~ms}$ ), which were measured off-line, the algorithm determines the correct time point to communicate with the hardware to initiate the stimulation. For instance, suppose at a given moment the algorithm initiates data fetch to populate the buffer with the last $5 \mathrm{~s}$ of EEG data, the data become available for processing a few milliseconds (say, $6 \mathrm{~ms}$ ) into the future based on sampling from the distribution for data fetch latency. Then, say it takes $100 \mathrm{~ms}$ for data processing to predict the next up state, which happens to be $600 \mathrm{~ms}$ into the future from the starting time point. If it takes a few milliseconds (say, $7 \mathrm{~ms}$ ) to physically initiate stimulation based on sampling from the distribution for stimulation command latency, the algorithm would wait $487 \mathrm{~ms}$ after the EEG processing step to send the stimulation command to the device. Ideally, tACS is applied for 5 cycles at the detected SW frequency over bilateral frontal electrodes (F3 and F4) at $1.5 \mathrm{~mA} /$ hemisphere with temporal/mastoid returns. Should processing times push beyond any potential stimulation time point, the algorithm compares the current time to the (now deprecated) stimulation time, and checks whether at least $300 \mathrm{~ms}$ of up-state stimulation is still possible. If so, the stimulation is initiated with an altered start phase (based on the detected SW frequency) for aligning with the endogenous SW oscillations, despite the delay, and is continued until 4 full cycles are completed (where a cycle is defined as the progression from $0^{\circ}$ phase to $360^{\circ}$ phase). In the event that at least $300 \mathrm{~ms}$ of up-state stimulation is not possible, then the algorithm plans the stimulation to start at the next upcoming up state based on the continued sine wave projections from the buffer. Once stimulation is delivered, the system idles for $3 \mathrm{~s}$ to avoid the collection of stimulation artifacts in the data buffer, then resumes the cycle of data update in the buffer, data processing and predictions, and stimulation planning as the criteria specified above are met. Our closedloop system thus adapts and adjusts stimulation parameters on-line to ensure the proper administration of stimulation at the correct temporal targets for matching the predicted transient brain states of interest. It is able to minimize the pitfalls of temporal inaccuracies that arise as a result of variable delays intrinsic to any recording/stimulation/processing hardware. On sham nights, up states were similarly predicted but no stimulation was applied.

\section{Experimental procedures}

The experiment was conducted over the course of $6 \mathrm{~d}$, including 3 nights spent in our sleep laboratory referred to here as "acclimation," "Night 2," and "Night 3," two afternoon follow-up testing sessions ("Day 2 Post-Sleep" and "Day 3 Post-Sleep"), as well as an initial orientation session. Participants were randomly assigned to one of the following four conditions in a withinsubjects, counterbalanced, single-blind design: Object Target/ Sham Stimulation Night 2, People Target/Active Stimulation Night 3 (SO/AP); Object Target/Active Stimulation Night 2, People Target/Sham Stimulation Night 3 (AO/SP); People Target/ Sham Stimulation Night 2, Object Target/Active Stimulation 
Night 3 (SP/AO); and People Target/Active Stimulation Night 2, Object Target/Sham Stimulation Night 3 (AP/SO). At the orientation session, participants were invited to provide informed consent, and were given several questionnaires to assess various aspects of their personality and sleep habits, as well as to gather an IQ estimate. Following the questionnaires, head measurements were made (circumference, nasion to inion, and preauricular to preauricular) to fit an EEG cap. Participants were next given a tour of the sleep laboratories and an explanation of the EEG/ stimulation equipment and experimental procedures. Finally, each participant was issued a Fitbit wrist-worn biometric sensor (Dickinson et al., 2016), with instructions on how to correctly operate it to track sleep before their laboratory visits.

For the acclimation night, participants arrived at the sleep laboratory by 5:00 P.M., and were prepped and fitted with an EEG cap (see Waking EEG data collection section), and an adapted version of Raven's Progressive Matrices, called Sandia Matrices, was administered (Matzen et al., 2010). Next, data were collected to calibrate biometrics for use in a predictive computational model, including a breath count task to measure attentional lapses (Braboszcz and Delorme, 2011) that lasted $30 \mathrm{~min}$, as well as a three-back task to generate cognitive stress and mental fatigue (Hopstaken et al., 2015) that lasted 21 min was gathered. Participants could then relax in the laboratory until approximately 9:00 P.M., when they were prepped for PSG recording during sleep (see Polysomnographic data collection section). EEG electrode locations were digitized using Polhemus FASTRAK System for data analysis purposes as well as to measure how much the cap may have shifted during the subsequent sleep episode. Participants were instructed to lie down in a supine position at approximately 10:00 P.M., when biocalibrations were performed to help identify sources of noise in later EEG acquisition. This included EEG data collection of eyes open for $1 \mathrm{~min}$, closed for $1 \mathrm{~min}$, looking up, down, right, and left, blinking slowly five times, clenching the jaw, and finally moving into a comfortable sleeping position. Lights out for the participants occurred between 10:00 P.M. and 11:00 P.M., and they slept for up to 8 uninterrupted hours before being awoken. During sleep, EEG data were monitored, and the closed-loop prediction algorithm was started when 4 min of continuous, visible $\mathrm{N} 2 / \mathrm{N} 3$ sleep was observed by a trained research assistant. During the acclimation sleep, no stimulation was applied, but the information gathered from the closed-loop prediction algorithm was used to verify the SW relative power threshold of 0.2 for subsequent experimental nights for each participant. Upon waking, participants could use the restroom and were offered water and snacks. They filled out the Karolinska Sleep Diary (KSD; Akerstedt et al., 1994) to assess subjective sleep quality. Next, they completed a one-back task for 21 min to assess alertness, and then were disconnected from the EEG hardware and released.

For night 2 , subjects arrived at the laboratory at approximately 5:30 P.M. and were immediately set up for EEG data collection and tDCS stimulation. Participants were seated in front of the computer and instructed on how to respond to the stimuli, but were not given specific information about the nature of the hidden targets or any strategies with which to find them. First, participants performed two baseline runs, consisting of 60 images/ run, and made a binary response (target present/target absent) using the keyboard. Each baseline run lasted $\sim 8 \mathrm{~min}$, and no feedback was given regarding performance.

Participants then took a brief baseline mood questionnaire to help assess potential effects of tDCS on subjective mood. The mood questionnaire consisted of nine questions on a $0-5$ Likert scale. Items included feelings of nervousness or excitement, tiredness, confusion, sadness, degree of frustration, dizziness, nausea, degree of physical pain or discomfort, and ability to pay attention. After all questions were answered, the training portion of the target detection task was administered.

Participants completed three training runs, the first two of which (training blocks one and two) were $<30$ min of either low-active $(1.0 \mathrm{~mA})$ or sham $(0.1 \mathrm{~mA})$ tDCS stimulation, followed by one more run (training block three) immediately following the administration of tDCS. The training blocks differed from the testing blocks in that after each choice the participants were given audiovisual feedback using a short clip regarding the consequence of their decision. If the participant indicated "target present" and was correct, a short video depicting the mission progressing as planned was shown, with a voiceover praising the participant for choosing correctly. If the participant incorrectly indicated that a target was present, a voiceover chastised them for delaying the mission, or insulted them by indicating they were acting cowardly. If the participant correctly indicated that there was no target present, feedback was given that the mission was progressing as planned. If participants incorrectly indicated that no target was present when in fact there was one, a video showing the consequence of missing the target was shown. For example, another member of the participant's platoon was shot by a sniper or a Humvee was destroyed by an improvised explosive device. Further, a voiceover scolded the participant for missing the target and told them that members of their team had been killed. Each of the three training blocks consisted of 60 trials each and lasted $\sim 16$ min. Each image was presented for $2 \mathrm{~s}$ with an intertrial interval that varied from 4 to $8 \mathrm{~s}$. The audiovisual feedback did not provide specific details of the shape or location of the target object, but enough information was available from the test image and feedback movie that the participant could infer the type and general position of the target in the image.

Following the three training runs, two more test runs (testing blocks three and four, referred to as an "immediate test") were administered to gauge the immediate effect of tDCS on learning before sleep. Half of the stimuli used in the immediate test had been presented during training (repeated stimuli), while the remaining stimuli were similar in content and had the same types of targets, but had not been presented during training (generalized stimuli). Thus, memory for trained images and the generalization of the training to novel images could be examined separately. Following the final test block, participants were administered an exit mood questionnaire consisting of the same nine questions in the initial mood assessment, as well as a questionnaire probing the strategy the participants used to complete the task. Next, a new set of Sandia Matrices was administered, as was a Language History Questionnaire. Then participants could relax in the laboratory until approximately 9:00 P.M., when they were prepped for PSG recording during sleep (see Sleep polysomnographic data collection section). EEG electrode locations were digitized, and biocalibrations were performed. Lights out for the participants occurred between 10:00 P.M. and 11:00 P.M., and they were allowed again to sleep for 8 uninterrupted hours before being awoken. During sleep, EEG data were monitored, and the closed-loop stimulation intervention was started when 4 min of continuous visible N2/N3 sleep was observed, and allowed to run through the remainder of the night. Participants received either closed-loop tACS (1.5 mA/hemisphere) or sham tACS (no current) for the entire duration of sleep (for a description of the intervention, see Closed-loop transcranial alternating current stimulation during slow-wave sleep section), administered at bilateral frontal anodes 
A

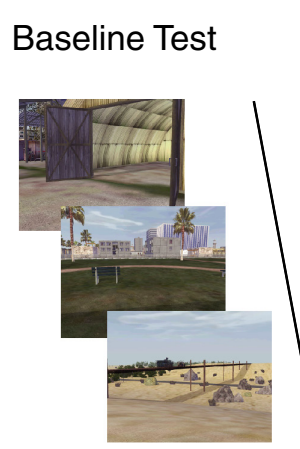

Respond:

Threat, No-Threat No Feedback

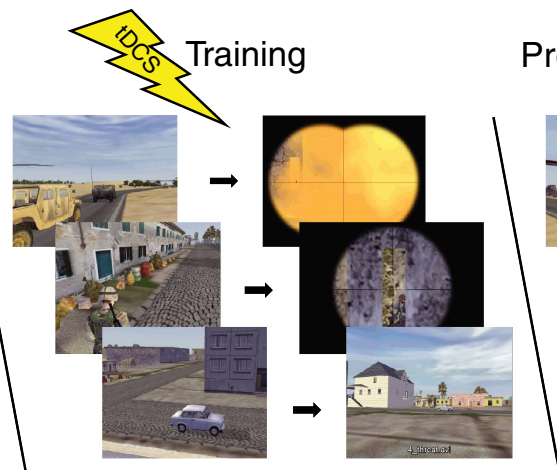

$1 \mathrm{~mA}$ anodal tDCS at F10, cathode left arm Respond: Threat, No-Threat

Video feedback on response accuracy
Pre-Sleep Test

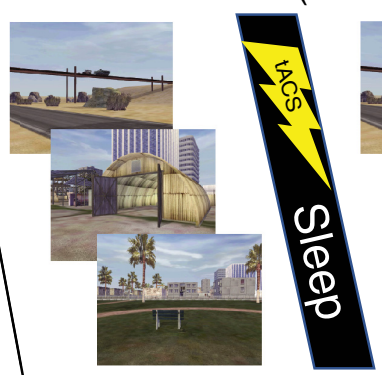

Respond:

Threat, No-Threat No Feedback
Post-Sleep Tests (Morning, Afternoon)

B
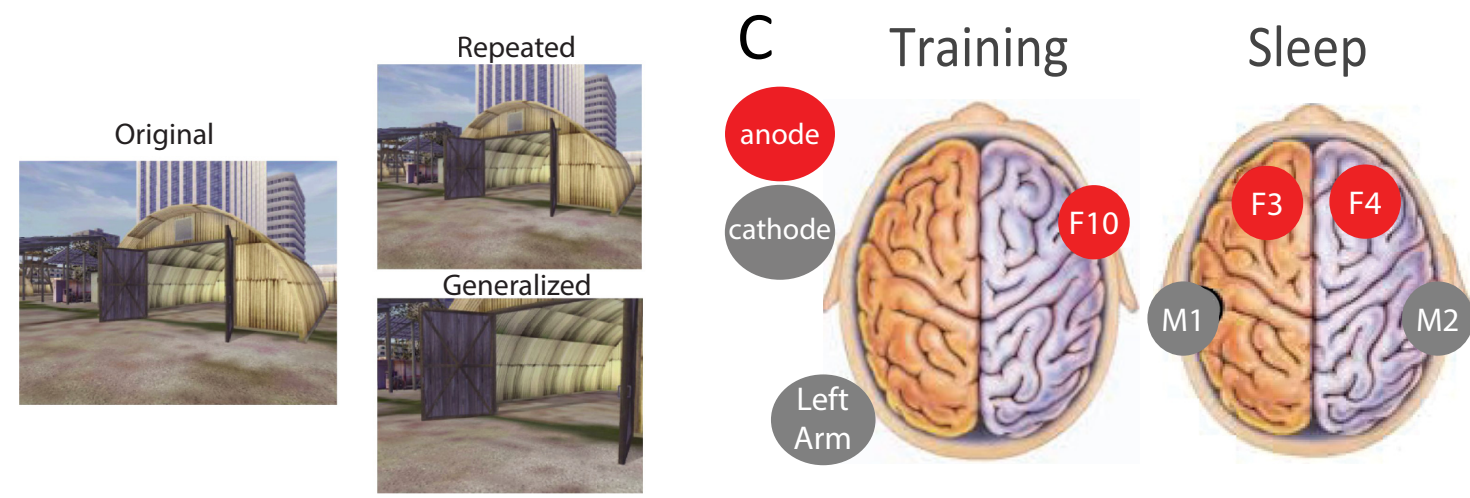

Figure 1. A, Target detection task. Participants identified cues of potential targets with binary target present/absent decisions. The study was conducted in multiple phases: baseline (i.e., no feedback on response), training (video feedbackidentifying correct and incorrect choices), immediate test (no feedback, immediately after training), and postsleep tests (no feedback, $\sim 12$ and $24 \mathrm{~h}$ after training). Active and sham stimulation conditions (counterbalanced within subjects) were administered at training (tDCS) and post-training sleep (closed-loop slow-wave tACS) with the stimulation montage shown in $\boldsymbol{C} . \boldsymbol{B}$, Memory was tested on two image types: repeated (identical from training to test) and generalized (same scene from training to test, but novel spatial viewpoint). $C$, tDCS montage used for training shown on left, and tACS montage used for slow-wave augmentation during sleep shown on right.

(F3 and F4 in the 10-20 system) and temporal/mastoid returns. These locations are based on previous slow-wave sleep interventions (Marshall et al., 2006; Westerberg et al., 2015; Ladenbauer et al., 2017). If the participant showed signs of waking, or needed to use the restroom, the stimulation was paused and resumed after the participant was again in N2/N3 sleep. Upon waking, participants were allowed to use the restroom and were offered water and snacks. They filled out the KSD to assess subjective sleep quality. Next, for the Day 2 Post-Sleep tests, they first completed two more testing blocks of the target detection task (referred to as the "morning test") to assess the effect of SW sleep augmentation on consolidation/performance, filled out the strategy questionnaire, and then were disconnected from the EEG hardware and released. For the second Day 2 Post-Sleep test, participants arrived $\sim 24 \mathrm{~h}$ after their initial Day 1 arrival (5:30 P.M.), were prepped for EEG data collection, and were administered two more testing blocks (referred to as the "afternoon test") to assess the effects of SW sleep augmentation on more long-term retention and performance. Note that each block of test runs (three through eight; i.e., immediate, morning, and afternoon tests) presented 60 repeated and 60 generalized images, and there was no overlap in stimuli across these runs.

Approximately $5 \mathrm{~d}$ after completing the Day 2 Post-Sleep tests, participants came back to the laboratory for their Night 3 and Day 3 follow-up. The timeline and procedures were identical to the Night 2 and Day 2 follow-up, the only differences being the target detection condition (object targets/people targets) and stimulation condition (active/sham) were the opposite of their Night 2 assignments. Upon completion of the Day 3 Post-Sleep tests, a final exit questionnaire was administered to gather subjective ratings from participants in terms of how they felt the intervention impacted their memory functioning generally, and they were debriefed, during which time they could ask questions about the nature of the experiment. Please see Figure 1 for a graphical description of the experimental procedures.

\section{Post hoc sleep EEG analysis}

Analysis of the sleep EEG data was performed using custom-built scripts implemented in Matlab R2016a (MathWorks) taking advantage of various FieldTrip (Oostenveld et al., 2011) and EEGLab (Delorme and Makeig, 2004) functions. EEG data were extracted from sleep sessions and epoched into prestimulation and poststimulation windows, which were in turn triggered by the SW oscillations described in the Materials and Methods section. The same process was carried out for the sham condition off-line by estimating where stimulation events would have occurred given the ongoing SW oscillations, and are synonymously referred to here as "SW events." It should be noted these sham SW events were computed post hoc on the full night's worth of data from the recording session. Indeed, the number of SW events significantly varied between active and sham conditions $\left(t_{(15)}=-8.01, p<0.001\right)$, with an average ( \pm SEM) of $280.87 \pm 45.58$ SW events on the active nights and $1223.70 \pm 84.32$ on the sham nights. An appreciable reason for the discrepancy is that the sham off-line code operates on the whole night without any starting latency or pauses, unlike the active on-line code 
where the intervention is initiated only after 4 min of continuous N2 sleep, and is paused in response to awakenings or any other visual disruption in the participants' sleep.

Pre-SW event epochs captured -6.4 to $-0 \mathrm{~s}$ before SW event onset, and post-SW event epochs captured 0 to $12.8 \mathrm{~s}$ relative to $S W$ event offset. Here, SW event offset occurs after the temporal duration of the targeted stimulation for that event, which is 5 cycles at the event-specific dominant frequency within the range of $0.5-1.2 \mathrm{~Hz}$ (i.e., a range from 4.16 to $10 \mathrm{~s})$. A segment-level artifact removal was performed within each epoch by searching in $200 \mathrm{~ms}$ sliding windows for a peak-to-peak voltage change of $500 \mu \mathrm{V}$ within each channel. Any segment that crossed this threshold was marked as bad and interpolated using non-artifactafflicted time points before and after the marked segments. Any channel that had $>25 \%$ of its segments within a given epoch marked as bad was discarded and the full epoch for that channel was interpolated using neighboring channels. Any SW event that had $>80 \%$ of its channels exceed the $25 \%$ segment threshold were discarded entirely. On average across channels, trials and subjects that were $15 \pm 22 \%$ (here, error is 1 SD) of prestimulation and $15 \pm 21 \%$ of poststimulation event epoch time points were interpolated in the active condition, and $3 \pm 4 \%$ of pre-SW events and $3 \pm 5$ post-SW events were interpolated in the active and sham conditions respectively. There were significantly more interpolated events in the active versus the sham condition (between-subjects $t$ test: pre-SW event, $t_{(15)}=2.56, p=0.02$; post-SW event, $t_{(15)}=2.48$, $p=0.03)$. The significantly noisier quality of the active EEG data also contributed to the discrepancy in SW events between active and sham conditions. After segment-level artifact removal, a pass of trial-level removal was performed such that any channel that exceeded the $500 \mu \mathrm{V}$ (minimum-to-maximum voltage change) threshold within a given SW event was reconstructed by interpolation of its neighbors, and any SW event in which $>80 \%$ of the channels exceeded that threshold the SW event was discarded entirely. Trial subselection was done with the constraint that each trial had at least $5.75 \mathrm{~s}$ of usable data both pre-SW and post-SW events. On average across subjects, $18 \pm 6 \%$ of sham and $19 \pm$ $28 \%$ of active SW events were discarded due to limited duration, and $10 \pm 8 \%$ of sham and $18 \pm 20 \%$ of active SW events were discarded due to $>80 \%$ of channels exceeding the artifact threshold. Following artifact removal, all epochs were truncated to -6.4 to $-1 \mathrm{~s}$ before the SW event and 3 to $12.8 \mathrm{~s}$ after the SW event to ensure that no stimulation artifacts lingered in the data. Finally, all epochs were mean centered, bandpass filtered between 0.1 and $125 \mathrm{~Hz}$, and bandstop filtered between 59 and $61 \mathrm{~Hz}$, and all channels were rereferenced to the global average across channels.

\section{Spectral power methods}

Time frequency decomposition was performed in FieldTrip using Morelet wavelets. Before decomposition, symmetric (mirror) padding was used to extend the pre-SW and post-SW event time-series to avoid edge artifacts in frequency decomposition. The series of wavelets used in the decomposition started with a width of 4 at the center frequency of $0.5 \mathrm{~Hz}$, and subsequent center frequencies were chosen such that each wavelet was $1 \mathrm{SD}$ in frequency domain from the previous wavelet. Simultaneously, the wavelet width was increased as a function of center frequency to minimize the combined uncertainty in time and frequency domains, with a starting width of 4 and maximum width of 12 . This yielded a time frequency representation with 52 approximately log-spaced frequency bins from 0.5 to $100 \mathrm{~Hz}$, and equally spaced time bins of $20 \mathrm{~ms}$. Normalized power within each frequency bin was calculated by first $z$-scoring within each SW event based on a mean and SD in power estimated over the whole time period ( -6.4 to $12.8 \mathrm{~s}$ ). Relative power within each frequency bin was then calculated using a baseline period across SW events by concatenating -3.5 to $-3 \mathrm{~s}$ from all pre-SW event periods and estimating a mean and SD from this concatenated time series. These values were then used to $z$-score within frequency bins both the before and after periods for each SW event to avoid single-trial bias in spectral normalization (Ciuparu and Mureşan, 2016). This $z$-scored change in power was then averaged across epochs within the active and sham stimulation conditions separately to yield a single channel $\times$ time $\times$ frequency matrix for each condition and subject.

\section{Phase-amplitude coupling methods}

Phase-amplitude coupling was estimated using an adaptation of the oscillation triggered coupling (OTC) method as described by Dvorak and Fenton (2014). OTC is essentially an event-related potential (ERP) analysis where the events are time locked to the peaks of high-power events within a targeted frequency band. Summing the voltage signal that is time locked to these events results in a waveform where the peak-to-peak amplitude (referred to as modulatory strength) can be used as a measure of how consistently the higher-frequency triggering events fall at a particular phase of the modulating lower-frequency signal. This method was chosen as it allows for estimates of coupling within relatively small window sizes (e.g., $<2 \mathrm{~s}$ ). This allowed for a more nuanced temporal analysis that was necessary given the dynamics of the spectral power results.

To estimate the influence of tACS on the relationship between the SW phase and the amplitude of spindle oscillations, the temporal onset of high-power events within the range of $8-16 \mathrm{~Hz}$ were determined using the same variable-width wavelet decomposition methods described in the Spectral power methods section. Here, approximately log-spaced frequencies from 8 to $16 \mathrm{~Hz}$ were estimated from 3 to $10 \mathrm{~s}$ every $2 \mathrm{~ms}$ to provide the most temporal precision possible given the sampling rate. The spectral estimates were then $z$-scored over time within each channel $\times$ frequency bin, and peaks within these bins that exceeded a $z$-score of 2 were used as triggering events. To differentiate where the strongest effects occurred in time, a sliding window with a $1 \mathrm{~s}$ duration and a separation (i.e., slide) of $0.25 \mathrm{~s}$ was used to segregate triggering events to a given temporal extent. In total, 21 overlapping windows were used, starting from 3.5 to $4.5 \mathrm{~s}$ and ending at 8.5-9.5 s relative to SW event offset. Here our hypothesis was that SW stimulation increased spindle coupling, therefore the voltage signal centered on triggering spindle events was filtered using a phase correcting second-order Butterworth filter with passband between 0.5 and $1.2 \mathrm{~Hz}$, as this was the range of frequencies used in tACS. Finally, a $1 \mathrm{~s}$ window centered on each of the triggering events for a given channel, modulated frequency, and the temporal extent were summed together to determine the underlying modulating signal, which was then characterized by taking the minimum-to-maximum amplitude referred to here as the "modulatory strength." This yielded a channel $\times$ modulated frequency $\times$ time matrix for each condition and subject. This measure of modulatory strength was $z$-scored based on an estimate of chance coupling within each channel $X$ modulated frequency $\times$ time bin. Here, the same number of triggering events for a given modulatory strength estimate were randomly sampled in time to produce an estimate of random coupling within that set of data. This process was repeated 200 times for each modulatory strength estimate to determine the mean and SD by which it was Z-scored.

Additionally, the preferred phase for a given channel $\times$ modulated frequency $\times$ time bin was estimated by collecting the phase angle from the SW filtered voltage signal centered on each triggering event. The average over this distribution was considered the preferred phase for each bin in the modulatory strength matrix of each subject and condition.

\section{Experimental design and statistical analyses}

Behavioral performance. To assess waking tDCS-related changes in performance a 2 (stimulation: active, sham) by 2 (image type: generalized, repeated) ANOVA with a dependent variable of F1 score in the target detection task was tested as a linear mixed-effects model using the lme4 package for R (Bates et al., 2015; R-Core-Team, 2015), and $p$ values for each predictor were estimated using the lmerTest package (Kuznetsova et al., 2015). Similarly, to investigate overnight changes in performance a 3 (test session: immediate, morning, afternoon) by 2 (stimulation: active, sham) by 2 (image type: generalized, repeated) ANOVA with a dependent variable of $\mathrm{F} 1$ score in the target detection task was tested as a linear mixed-effects model using the lme4 package for R. This model allowed us to account for the random effects related to individual subjects while also allowing us to include the four subjects who had missing data for a single post-sleep test session (2 missing active-afternoon, 1 missing shammorning, and 1 missing sham-afternoon). Test session contrasts were coded to compare the average F1 score in the post-sleep tests to the immediate test (i.e., immediate $<$ mean(morning, afternoon), referred to as "postsleep vs presleep"), and a linear increase with time (i.e., imme- 
diate $<$ morning $<$ afternoon, referred to as "temporal increase"). For the overnight model, subjects were used as a random factor for the main effects of stimulation and image type (excluded effects from the full random model did not significantly contribute, $\chi^{2}(4)=4.25, p=0.37$ compared with the full model), and fixed effects were defined for the full three-way interaction of test session by stimulation by image type. A similar model was also used for the tDCS effects, with subjects as random factors for the main effects of stimulation and image type. Results from these models are reported here as significant where any predictor reached an $\alpha$ level of $p<0.05$.

Targeted up-state validation. A test for the precision of the up-state detection algorithm was performed using a V-test on the distribution of phase angles within each subject, as well as the distribution of average phase for each subject across the group (Berens, 2009). The null hypothesis of this test was the phase within each subject is either uniformly distributed or not reliably different from the targeted phase of 0 .

A test for the distribution of algorithmically detected SW events per sleep stage was performed using a linear mixed-effects model with the percentage of total SW events overnight broken down by sleep stage as the dependent variable, a fixed-effect categorical variable identifying individual sleep stages, and a stimulation condition grouped by subjects as a random factor. All but one session were included in this analysis; a single subject's active night sleep stage data were missing and could not be accounted for. Here, three specific contrasts were tested to determine whether detected SW events were biased to stages N2 and N3. The first compared the average in $\mathrm{N} 2$ and $\mathrm{N} 3$ with the average over all other sleep stages. The second compared counts in N2 to counts in REM, and the third compared N3 to REM.

Spectral power and modulation strength. Significant changes in relative power and correlations with behavior were estimated using the FieldTrip permutation-based clustering algorithm (Maris and Oostenveld, 2007). A contrast of the relative change in power (post-SW event normalized by pre-SW event, as described in the EEG methods section), for the active stimulation condition compared with the relative change in power for the sham condition was made for each channel $\times$ time $\times$ frequency bin between 3 and $10 \mathrm{~s}$ from offset of SW events. These contrasts were evaluated using a paired $t$ test over subjects, and a cluster-based permutation test was performed to determine the significant channel $X$ time $X$ frequency bins. Clusters were created by grouping adjacent bins that had an $\alpha$ level of $p<0.05$. Each cluster was then characterized by the sum of its $t$ values, and a surrogate distribution of clusters, similarly characterized, was created by shuffling the subject labels and repeating the clustering procedure 500 times. Thus, a clusterwise significance value can be attributed to each observed cluster in reference to its position in the permutation-based surrogate distribution. Here we report any cluster that reached a clusterwise significance of $<0.05$ (i.e., $95 \%$ of the surrogate clusters had smaller summed $t$ values then the observed cluster). Any contrast cluster that reached this clusterwise threshold was then used as a mask to perform a subsequent cluster-based permutation test on the correlation between behavior and the significant channel $\times$ time $\times$ frequency bins. This effectively limits the correlation cluster analysis to the channel $\times$ time $X$ frequency bins that a priori show a significant difference between the active and sham stimulation conditions. Behavioral measures correlated with this masked change in relative power were limited to the measures that showed a significant difference between active and sham conditions, which included an F1 score on generalized images alone and the interaction of generalized versus repeated images. A separate cluster analysis was performed for each of these behavioral measures where correlation coefficients for each significant channel $\times$ time $\times$ frequency bin was calculated within each subject, transformed into a $t$ value, and adjacent bins that had a significance of $p<0.05$ were clustered together. The same permutation-based significance test was performed as in the contrast clusters, where a surrogate distribution of clusters was created by shuffling the subject labels and repeating the correlation clustering procedure 500 times. This number of permutations at the $\alpha$ level of 0.05 leads to an expected error of \pm 0.01 in the clusterwise $p$ values. This hierarchical clustering procedure focuses on extracted biomarkers that account for performance differences between active and sham conditions and are induced by closed-loop tACS, compared with biomarkers that are agnostic to brain stimulation.

Significant modulation strength (of phase-amplitude coupling between slow waves and spindles) and its relationship to behavior was determined using a masked cluster-based permutation test similar to the method used for spectral power. In this analysis, the first-level contrast is between active and sham conditions using data in the $z$-scored modulation strength matrix. Clusters of channel $\times$ modulated frequency $\times$ time bins that reach a clusterwise $p<0.05$ are then passed onto to the masked correlation clustering. Here we again restricted behavioral measures to F1 score on generalized images and the interaction of generalized and repeated images.

A test of the significance of a preferred phase for OTC waveforms was performed on the distribution of phase angles using Rayleigh's test for nonuniformity with no specific hypothesized mean direction.

\section{Results}

\section{Behavioral results}

tDCS-related changes

As shown in Figure $3 A$, active tDCS stimulation showed no significant difference in performance compared with the sham condition $\left(t_{(32.9)}=0.881, p=0.385\right)$, and there was no difference in performance for the repeated versus generalized images $\left(t_{(29.18)}=\right.$ $-1.067, p=0.295)$. There was a marginally significant interaction between stimulation and image type $\left(_{(29.35)}=-1.90\right.$, $p=0.067$ ) such that repeated images show an increase in performance for active versus sham tDCS (mean \pm SEM: active $=$ $0.81 \pm 0.04 ;$ sham $=0.83 \pm 0.04)$, while the generalized images show the reverse (active $=0.74 \pm 0.03$; sham $=0.78 \pm 0.03$ ). To address any concerns regarding outlier subjects, an estimate of each subject's influence on the model was performed using Cook's distance. A single subject had a Cook's distance that exceeded 2 SDs from the mean distance across subjects. This subject was removed from the model; however, there was no substantive change in the pattern of results. In general, these results suggest that there was no reliable effect of tDCS on performance, and, if anything, tDCS improved repeated image performance more so than generalized image performance.

\section{Overnight changes in behavior}

A significant main effect for image type $\left(t_{(44.36)}=-2.74, p=\right.$ 0.008 ) was found, implying that generalized images, averaged across test sessions and stimulation conditions, had a lower F1 score compared with repeated images. Critically, however, the postsleep versus presleep by stimulation by image type interaction $\left(t_{(143.76)}=2.451, p=0.015\right)$ and the temporal increase by stimulation by image type interaction $t_{(143.76)}=2.457, p=0.015$ ) were both significant. Again an estimate of outliers was performed using Cook's distance. Again, the same subject as the tDCS-related changes was determined to be an outlier with a Cook's distance of $>2$ SDs for the group, and, again, rerunning the model without this subject yielded no substantive change to the pattern of results. This suggests that the change in F1 score after sleep is greater for the active compared with sham stimulation conditions, and this relationship is contingent upon the test image type being generalized rather than repeated. A follow-up $t$ test comparing the postsleep versus presleep change in F1 score on generalized images for active versus sham conditions also showed a significant difference $\left(t_{(15)}=2.79, p=0.014\right)$. These relationships are illustrated in Figure $3 B$. More detailed behavioral results are presented in Jones et al. (2018). 

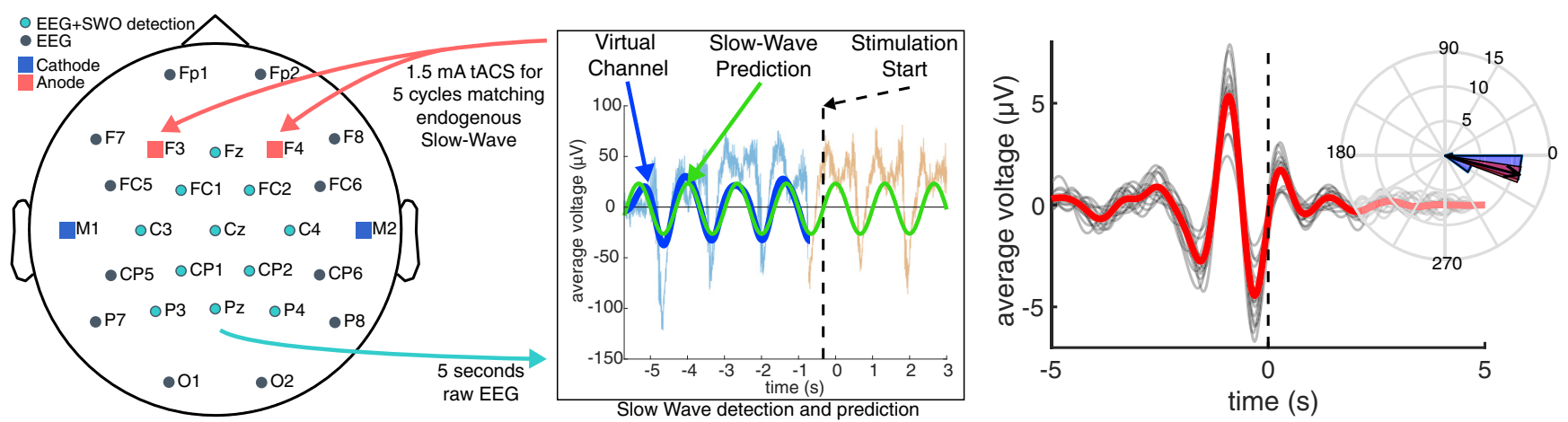

Figure 2. Illustration of the closed-loop system. The left plot shows a standard 10-20 electrode montage used during closed-loop tACS, and the middle plot shows an example of slow-wave detection and prediction from a given subject's sham session (time locked to the peak of the targeted up state). Raw EEG from the teal electrodes shown on the left are minimally preprocessed, then averaged together to form a single virtual channel used for slow-wave detection (shown in light blue). This signal is filtered between 0.5 and $1.2 \mathrm{~Hz}$ (shown in dark blue), then used to produce a predictive sine wave fit (shown in green) that is used to schedule the next up state (shown with the dashed line). The light orange line shows the unfiltered virtual channel outside the predictive buffer only available during sham sessions. The right plot shows a group-level ERP from sham nights centered on the start of the predicted up states. Red line shows average bandpass-filtered voltage in the slow-wave band $(0.5-1.2 \mathrm{~Hz})$ over the within-subject averages shown in gray. The inset plot is the average phase angle at the start of the predicted up state across subjects. Blue-shaded regions show the histogram over 16 subjects, the black arrow is average over those subjects, and the red-shaded region shows $95 \%$ confidence around the mean.

\section{EEG Results}

Slow-wave up state-targeted stimulation

Validation of closed-loop targeted stimulation was done off-line using sleep EEG data from sham nights to get an artifact-free estimate of the temporal accuracy of the up-state predictions during the active nights and also to generate SW event markers for sham nights for use in post hoc EEG analyses. EEG data were extracted in $10 \mathrm{~s}$ windows surrounding the start of each predicted up state as part of the off-line code to mark sham SW events. Because of the stochastic delays in the system related to data fetch, processing, and stimulation planning operations, the stimulation does not always start at the zero phase of the predicted next up state. The majority of the stimulation events would be initiated during the first up state or at the start of the second up state of the predicted sine wave fit to the endogenous SW oscillations. Therefore, useful averages of consistent phase cannot be made with extracted epochs centered at the start of actual stimulation. To address this, epochs were centered at the start of the first predicted up state, filtered in the SW band from 0.5 to $1.2 \mathrm{~Hz}$ using a phase-corrected second-order Butterworth filter, Hilbert transformed, and again phase corrected by $90^{\circ}$ to align with the sine wave prediction used in the closed-loop system. The average phase at the start of the first predicted up state across events within each subject was calculated and tested against 0 (i.e., the intended phase for the start of the positive half waves of the SW oscillations). Using a V-test for circular uniformity (Berens, 2009), each subject rejected the null hypothesis illustrating that the phase within each subject is both nonuniformly distributed and not different from the targeted phase of 0 . These average phase estimates are then aggregated across subjects in the polar histogram shown in Figure 2, and the average ERP across events centered at the targeted up-state onset is plotted for each subject as gray lines, with the average over subject ERPs overlaid in red.

Because the closed-loop intervention was allowed to deliver stimulation whenever a SW oscillation reached a sufficient relative amplitude, we assessed the distribution of stimulation events across sleep stages in a post hoc analysis, as illustrated in Figure 3. Here, collapsed across active and sham conditions, the percentage of total SW events for a given night averaged over stages N2 and N3 was larger than the average percentage of SW events over all other stages $\left(t_{(213)}=14.4, p<0.001\right)$. Further, the percentage counts in N2 $\left(t_{(213)}=10.82, p<0.001\right)$ and N3 $\left(t_{(213)}=3.58, p<\right.$ $0.001)$ were significantly larger than that in REM.
Stimulation-induced spectral power changes

Post-SW event changes in power were estimated between the active and sham conditions using the clustering procedure described in the Experimental design and statistical analyses section; in short, the contrast of relative post-SW event power in the active versus sham conditions were clustered between 3 and $10 \mathrm{~s}$ poststimulation across all channels and frequencies. Three clusters reached a clusterwise threshold of $p<0.05$. The first cluster is a positive cluster (i.e., active greater than sham) shown in Figure $4 A$, with a temporal extent from 3.02 to $4.22 \mathrm{~s}$ relative to stimulation offset, a frequency extent from 0.5 to $4.7 \mathrm{~Hz}$, and a clusterwise $p$ value of 0.036 . The second cluster is a negative cluster, shown in Figure $4 B$, with a temporal extent from 4.28 to $9.88 \mathrm{~s}$, a frequency extent from 0.5 to $47.2 \mathrm{~Hz}$, and a clusterwise $p$ value of 0.008 . The third cluster is a negative cluster, with a temporal extent from 4.62 to $9.82 \mathrm{~s}$, a frequency extent from 0.5 to 2.6 $\mathrm{Hz}$, and a clusterwise $p$ value of 0.047 (this cluster yielded no significant correlations with behavior and is therefore omitted from plots).

\section{Behavioral correlations with stimulation-induced spectral power changes}

A similar cluster analysis was performed when correlating overnight differences (postsleep - presleep) in F1 score with power changes. However, this analysis was restricted by using the significant contrast clusters as masks to subselect the channel $\times$ time $\times$ frequency bins used in the correlation with the behavior change differences. Separate correlation cluster analyses were performed using each of the significant contrast clusters as a mask and correlating with F1 score changes from the generalized images, as well as the interaction between generalized and repeated images. In total, this yielded six follow-up tests.

Correlating the difference in overnight F1 score in the generalized images with each of the significant contrast clusters yielded a significant positive correlation cluster only when using the positive contrast cluster, shown in Figure $4 A$, as a mask. The correlation cluster, shown in Figure $5 A$, had a very similar temporal extent as its mask from 3.02 to $4.14 \mathrm{~s}$, a more restricted frequency extent from 0.5 to $2.0 \mathrm{~Hz}$, and a clusterwise $p$ value of 0.002 .

Correlating the difference in overnight F1 score changes for generalized images versus repeated images yielded significant positive correlations when using both the positive (Fig. 4A) and negative (Fig. $4 B$ ) contrast clusters as masks. The positive con- 
A
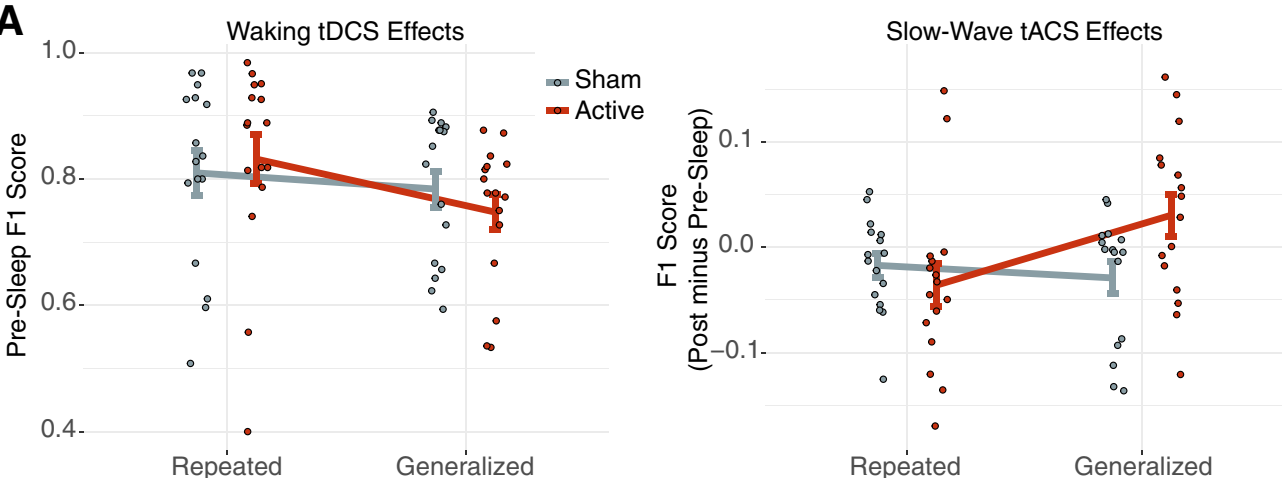

B
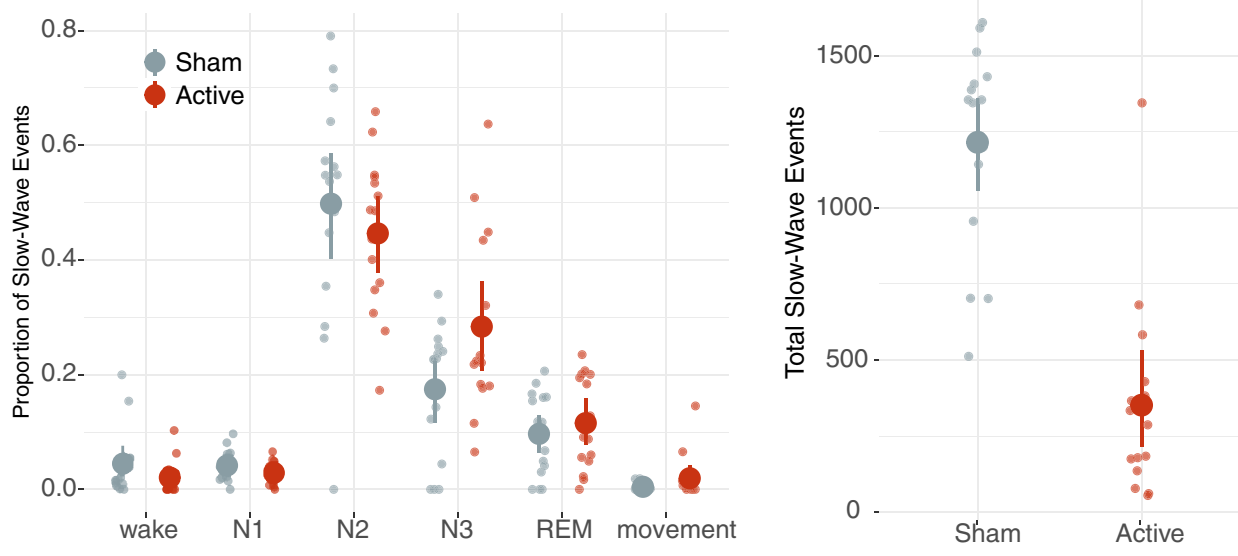

Figure 3. A, Left plot, The behavioral effects from waking $\mathrm{tDCS}$. Target detection F1 score from the test immediately following training plotted as an interaction of image type by stimulation condition. No significant main effects were observed, and a marginal interaction was found. The right plot shows overnight closed-loop slow-wave tACS effects. Here the change in F1 score from the average postsleep - presleep performance is shown as an interaction between image type and stimulation condition. Critically, a significant interaction between image type and stimulation condition showed an increased overnight change in F1 score for generalized images in the active condition. Each dot is a given participant's performance, and error bars show the SEM. $\boldsymbol{B}$, Left plot, Detected SW events broken down per sleep stage as a proportion of the overnight total for each subject and condition. Here stages N2 and N3 on average had more SW events than all other stages. Right plot shows total SW events for each stimulation condition. Here active stimulation had fewer total SW events compared with sham. Small dots reflect individual subjects, large dots reflect the group mean, and error bars are boot-strapped $95 \%$ confidence intervals.

trast cluster mask yielded a significant positive correlation, shown in Figure $5 B$, with a similar temporal extent compared with its contrast cluster mask from 3.02 to $4.06 \mathrm{~s}$, a restricted frequency extent from 0.5 to $0.8 \mathrm{~Hz}$, and a clusterwise $p$ value of 0.008 . The negative contrast cluster mask yielded a significant positive correlation, shown in Figure $5 C$, with a restricted temporal extent compared with its contrast cluster mask from 4.74 to $8.90 \mathrm{~s}$, a restricted frequency extent from 0.5 to $2.0 \mathrm{~Hz}$, and a clusterwise $p$ value of 0.034 .

\section{Phase-amplitude coupling}

Changes in phase-amplitude coupling were assessed using an adaptation of OTC with triggering frequencies from 8 to $16 \mathrm{~Hz}$ such that the triggered events were segregated into $1 \mathrm{~s}$ windows spaced every $0.25 \mathrm{~s}$, starting from 3.5 to $4.5 \mathrm{~s}$ and ending at 8.5 to $9.5 \mathrm{~s}$ relative to SW event offset. A comparison between active and sham modulation strength was made by calculating the minimum-to-maximum amplitude of the OTC waveform filtered in the SW band $(0.5-1.2 \mathrm{~Hz})$ for each channel $\times$ modulated frequency $\times$ time bin. Using these data, a cluster analysis of modulation strength was performed in a fashion similar to that of the spectral power changes.

As shown in Figure 6, the active stimulation condition showed a marginally significant increase in SW phase to high spindle (13-16 Hz) amplitude coupling in the time windows centered at 4 and $4.25 \mathrm{~s}$ (including all events from 3.5 to $4.75 \mathrm{~s}$ ), with a clusterwise $p=0.053$. This increase in active stimulation modulation strength highly overlaps in time and spatial topography with the stimulation-induced increase in SW power and its correlation with behavioral changes. The preferred phase of the SW modulating signal in the significant channel $\times$ modulated frequency $\times$ time bins for the active condition was nonuniformly distributed across events (Rayleigh's test for nonuniformity, $r=$ $7.78, p<0.001$ ) and centered at $262^{\circ}$. The preferred phase in the sham condition was not significantly different from a uniform distribution $(r=0.03, p=0.85)$.

Two other clusters, not shown in the figures, were found with significant decreases in coupling for the active compared with sham conditions. The first spanned from 9 to $16 \mathrm{~Hz}$ and from 6.25 to $7.25 \mathrm{~s}$ (including all events from 5.25 to $8.25 \mathrm{~s}$ ), with a clusterwise $p=0.02$. The other spanned $13-16 \mathrm{~Hz}$ and from 8.5 to $9.0 \mathrm{~s}$ (including all events from 8 to $9.5 \mathrm{~s}$ ), with a clusterwise $p=0.03$. Both clusters showed a predominant spatial topography over the right central-posterior electrodes and no significant preferred phase.

A follow-up masked analysis for behavioral correlation was also performed using each of the significant clusters found in active versus sham modulation strength; however, no significant clusterwise correlation with behavior was found for any of the three masks with F1 score on generalized images or the interaction between generalized and repeated images. 

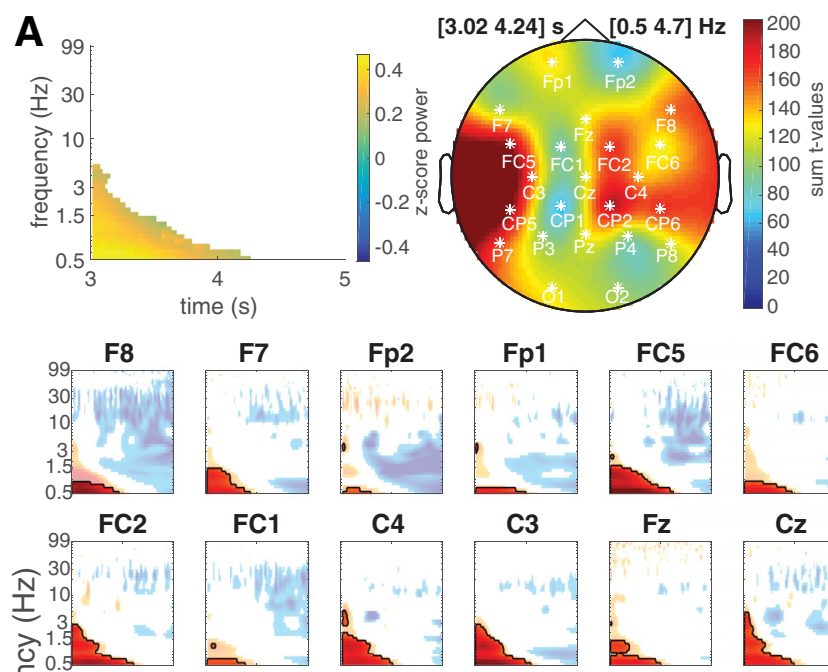

FC6
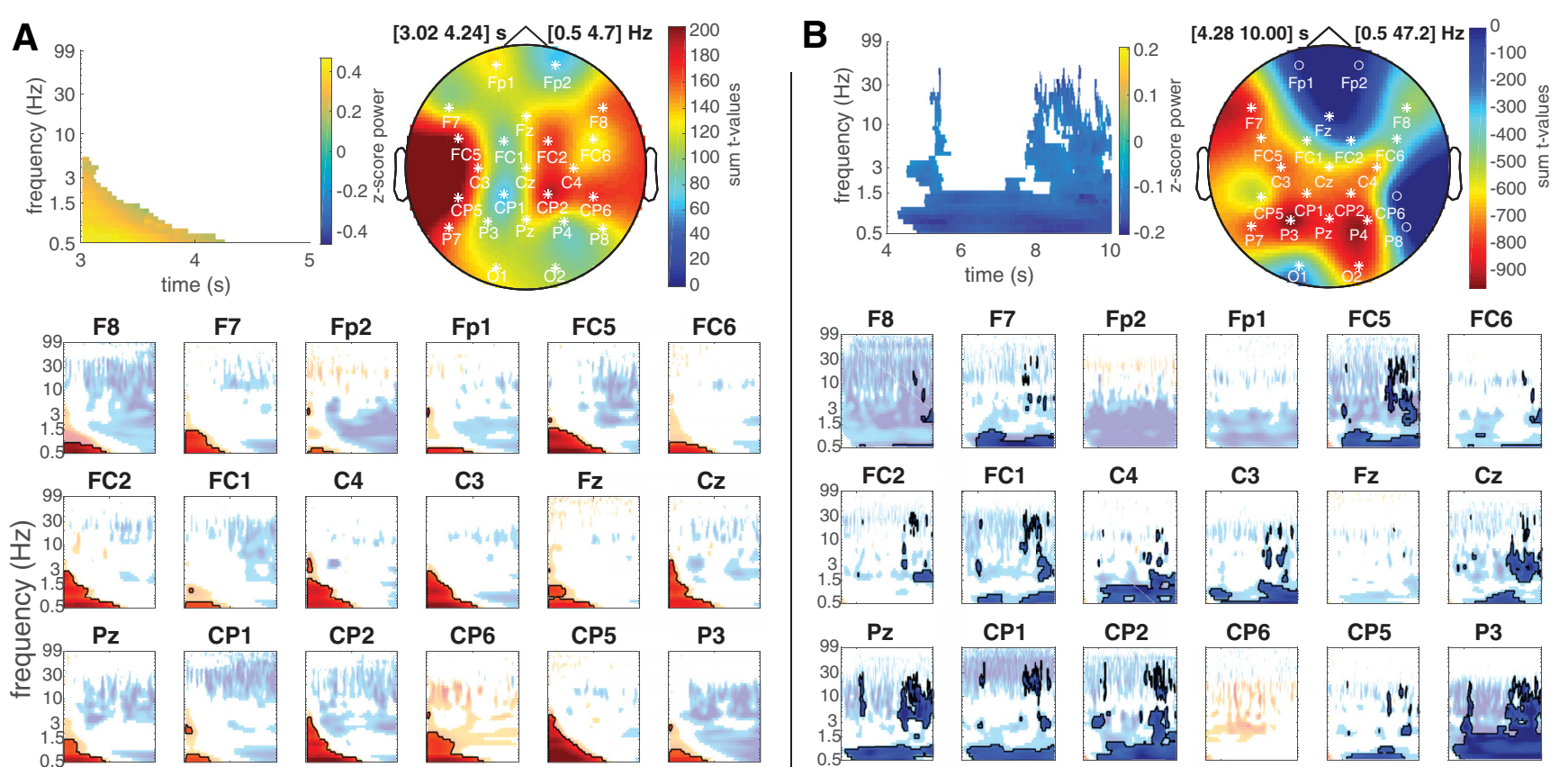

Fp2
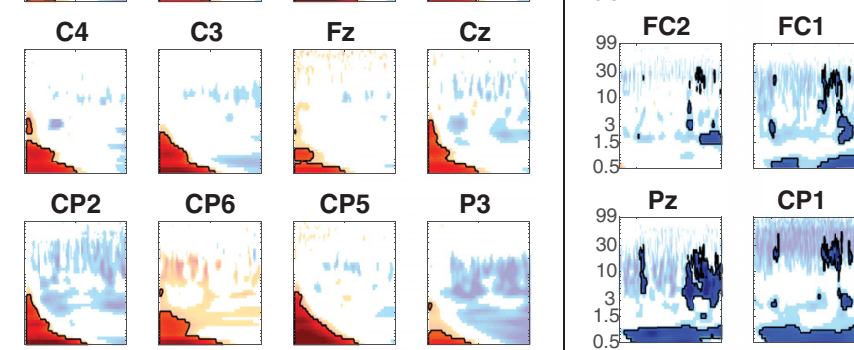

P7

P8
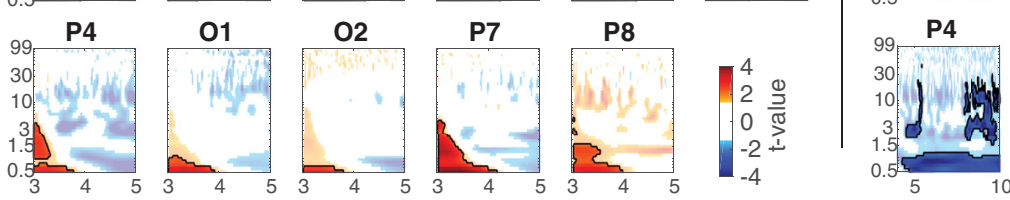

time (s)

Figure 4. Contrast of relative post slow-wave event power in the active versus sham conditions. $A$, Positive cluster showing active greater than sham (clusterwise $p=0.036$ ). Top left plot, Average power difference from significant channel $\times$ time $\times$ frequency bins. Top right plot, Topography of summed $t$ values from significant time $\times$ frequency bins. Bottom plot, $t$ values from significant time $\times$ frequency bins for each channel. Outlined areas show significant bins, and desaturated areas show nonsignificant bins, with an $\alpha p<0.05$. B, Negative cluster showing sham greater than active with clusterwise $p=0.008$. The plot layout is the same as in $\boldsymbol{A}$.

\section{Discussion}

We have shown that fully closed-loop tACS can be used to effectively target endogenous SW oscillations during natural sleep. This stimulation shows an increase in SW power as late as $4.2 \mathrm{~s}$ and is paired with a corresponding increase in coupling with high spindle power. Further, this increase in SW power correlates with behavioral changes in long-term memory performance generally consistent with theories of systems-level consolidation. Moreover we show evidence for a decrease in SW power and coupling with spindle amplitude in active stimulation relative to sham starting $\sim 5 \mathrm{~s}$ after stimulation offset. This decrease may be indicative of a SW refractory period induced by stimulation; however, these periods still show a positive correlation with behavior such that the longer the stimulation-induced power increase is maintained, the better the postsleep memory performance.

The use of a frequency agnostic clustering approach in this work adds to the validity of the results, as no a priori definition of frequency bands was incorporated into the analysis; however, known biologically relevant frequency bands manifested in the results. Similarly, the focus on stimulation-induced spectral changes through the use of a masked correlation cluster analysis narrows results to those that are related to the stimulation intervention. This not only adds more sensitivity, but allows for more specific interpretation of the changes witnessed. These results help build a better understanding of the potential for improving memory consolidation during sleep and address growing concerns related to the efficacy of tACS to induce physiological and behavioral changes.

\section{Waking tDCS versus sleep-dependent slow-wave tACS}

In the present study, no significant effect of tDCS was found with a $1.0 \mathrm{~mA}$ current dose; however, a nonsignificant difference in the appropriate direction was observed. It is possible that differences in details of current generation and control between the iontophoresis systems used previously and the current StarStim system may be involved. Because of this null effect and previous studies showing no overnight change in performance related to tDCS in this target detection task, it is likely that all overnight behavioral effects can be attributed to SW tACS.

Given this, it is unclear why there was no improvement in repeated images induced by SW tACS, as might be expected based on previous studies. Generally, most of these studies have seen effects in some form of a paired-associates task, either word pairs (Marshall et al., 2004, 2006; Westerberg et al., 2015), or visual object paired associates (Prehn-Kristensen et al., 2014; Ladenbauer et al., 2017). The target detection task, particularly for the repeated images, requires participants to find camouflaged targets in complex scenes, and therefore relies more on recognition of perceptual details compared with standard paired-associates tasks. This may explain why no overnight effects were found in the repeated images alone. In contrast, the strength of the generalized images in the target detection task is that the performance for a given test cue image is not determined by the best match to the originally studied cue image, but instead its relation to the scene originally studied and the potential for a target to exist in that scene. This provides a much more ecologically plausible memory-testing paradigm, allowing for very different percep- 

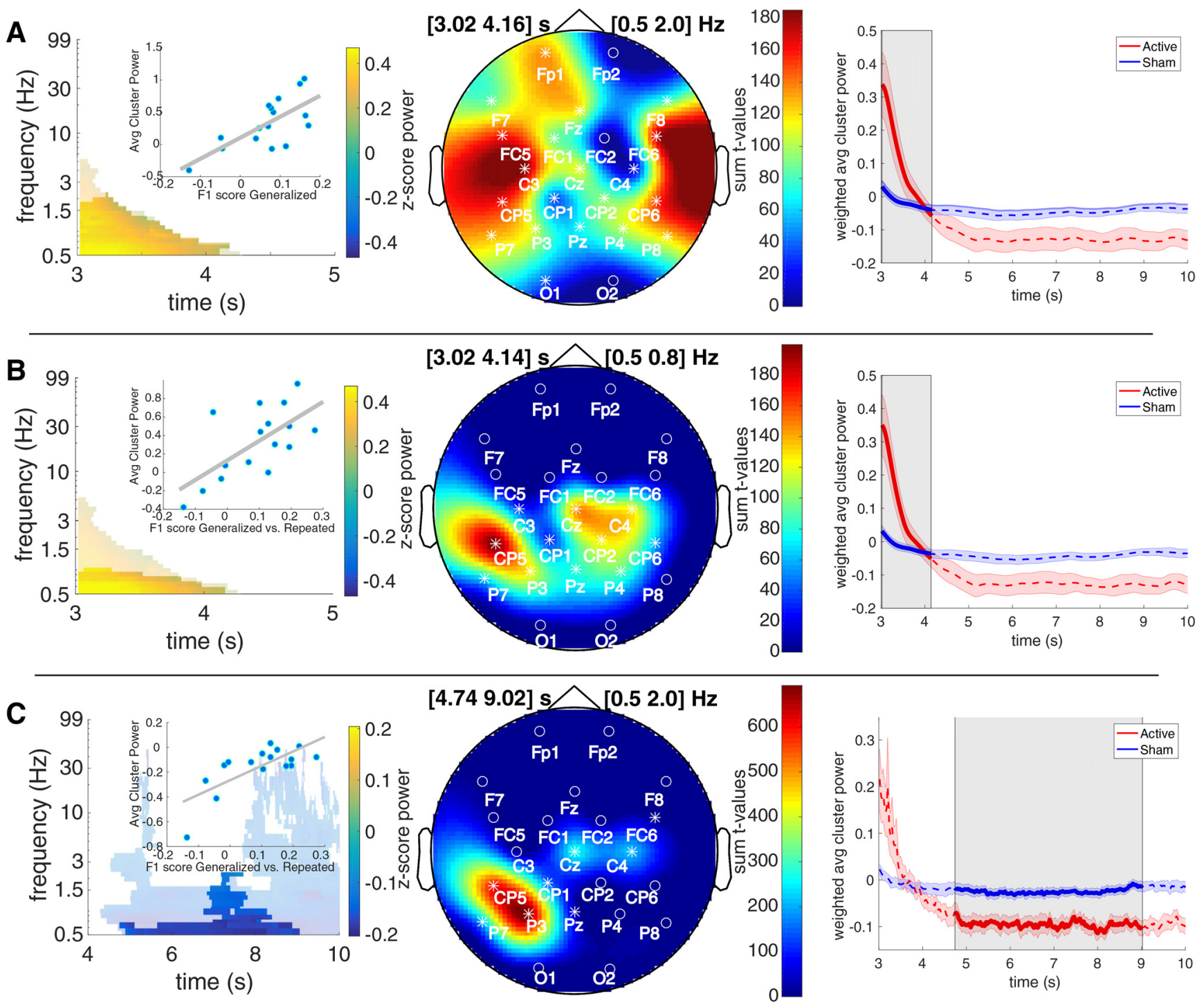

Figure 5. Significant $(p<0.05)$ masked correlation cluster results. $A$, Correlation of spectral power change difference from significant positive contrast cluster bins (Fig. 4A) and the overnight change (postsleep - presleep) in F1 score for generalized images, with a clusterwise $p=0.002$. Similar to the plot layout in Figure 4 , the left plot shows the average spectral power change difference between active and sham within the significant correlation cluster bins (desaturated area shows the nonsignificant bins from the full mask). Illustrative inlay of scatter plot based on average power change difference per subject over all significant bins correlated with the overnight change in $\mathrm{F} 1 \mathrm{score}$ for generalized images. Middle plot, Topography of summed $t$ values from significant time $\times$ frequency bins. Right plot, Time-series of weighted (by summed tvalues from significant correlation channel $X$ frequency $X$ time bins) average of active and sham power. Significant correlation cluster time points marked in dark-shaded region, and error ribbons are SEM. B, Correlation of spectral power change difference from significant positive contrast cluster bins (Fig. $4 A$ ) and the overnight change difference in F1 score on generalized images - repeated images, with a clusterwise $p=0.008$. The plot layout is the same as in $A$. C, Correlation of spectral power change difference from significant negative contrast cluster bins (Fig. $4 B$ ) and the overnight change difference in F1 score on generalized images - repeated images, with a clusterwise $p=0.034$. The plot layout is the same as in $\boldsymbol{A}$ and $\boldsymbol{B}$.

tual cues to imply the same underlying study item, which is ideal for testing the consolidation of essential gist rather than specific items.

\section{Stimulation-induced spectral power decreases}

The time period and broad frequency range that shows a decrease in SW power for active versus sham conditions suggests that there may be some stimulation-induced refractory period, and it is unclear how long this period of decreased power lasts. Masked correlation analysis shows that this decrease has the same relationship to behavior as the stimulation-induced increases in power shown in Figure 5, $B$ and $C$. This suggests that the stimulation-induced relationship with behavior is relatively constant throughout the analysis window; however, the induced power changes start at a level that is higher than the sham nights and slowly decreases over time.
Previous results have shown stimulation-induced decreases in SW oscillations. Ngo et al. (2015) applied auditory clicks in predicted up states to enhance endogenous SW oscillations and found that SW events and spindle-SW coupling decreased as the number of contiguous stimulation events increased. Interestingly, they found that this decrease did not negatively impact behavior. In reference to our results, this suggests that the stimulation-induced refraction in SW power is a normal self-limiting function, such that large increases in SW power as a result of stimulation are accompanied by periods of suppressed activity.

Previous sleep-dependent memory-enhancing tACS findings Several empirical results exist addressing the effectiveness of transcranial electric stimulation (tES; here encompassing both direct and alternating current) at inducing measurable electric 

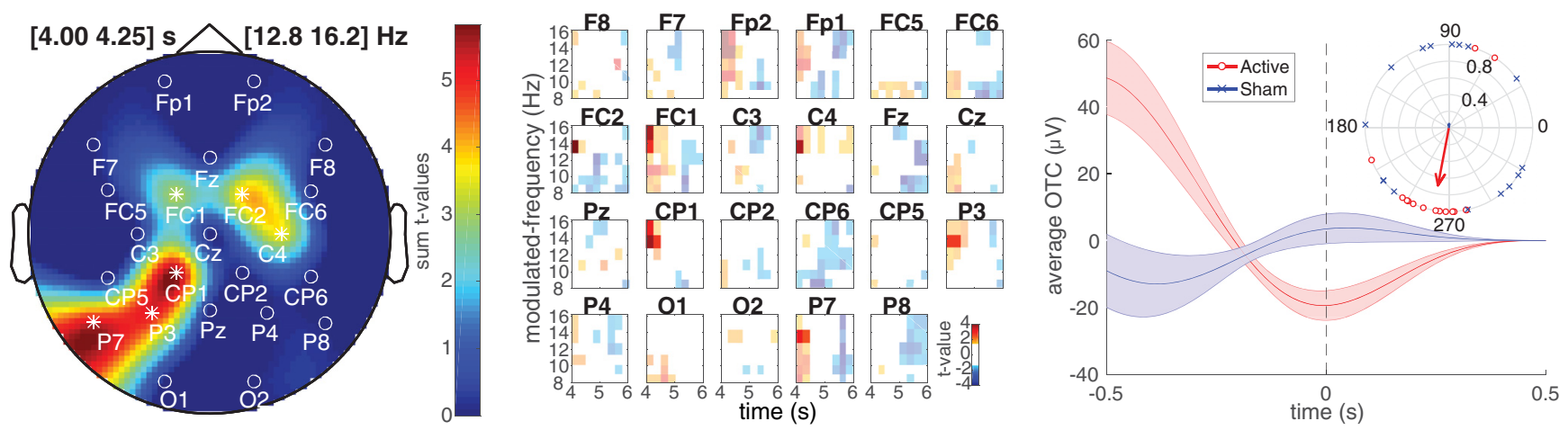

Figure 6. OTC-derived measures of phase-amplitude modulation strength (minimum-to-maximum measures of the average voltage signal temporally centered on high-power events within the modulated frequency, derived from $1 \mathrm{~s}$ windows centered on each temporal bin). Here, a marginally significant (clusterwise $p=0.053$ ) positive cluster shows active stimulation with greater slow-wave modulation strength of high spindle $(13-16 \mathrm{~Hz}$ ) band amplitude derived from spindle events occurring between 3.5 and $4.75 \mathrm{~s} \mathrm{relative} \mathrm{to} \mathrm{slow-wave} \mathrm{event} \mathrm{offset.} \mathrm{Left} \mathrm{plot,} \mathrm{Summed} t$ values from significant cluster bins. Middle plot, Individual $t$ values from the time $\times$ modulated frequency plots for each channel with the nonsignificant bins desaturated. Right plot, The average slow-wave filtered OTC waveform for active and sham conditions derived from significant cluster bins. These waveforms reflect the summed voltage centered on high-power modulating frequency events, which are then averaged over significant cluster bins, and finally over subjects; the ribbon is the SEM over subjects. The inset polar plot shows the average preferred phase for each subject in the active (preferred phase $=262^{\circ}, p<0.001$ ) and sham (no significant preferred phase) conditions.

fields within the brain (Horvath et al., 2015; Opitz et al., 2016; Huang et al., 2017; Lafon et al., 2017; Ruhnau et al., 2018), as well as several studies showing various behavioral changes beyond those targeted here (for review, see Jacobson et al., 2012). The main concerns surrounding the effectiveness of tES is that the induced electric fields are not sufficient to bias network-level firing patterns or oscillations, as is the assumed mechanism of tES protocols. Our approach, however, attempts to target ongoing endogenous oscillations, both in frequency and phase, as opposed to inducing them de novo. Based on in vivo studies, the estimated threshold of influence for induced electric fields when attempting to match endogenous activity is $0.25 \mathrm{~V} / \mathrm{m}$ (Jefferys et al., 2003; Reato et al., 2010; Lafon et al., 2017). Conversely, the measured maximal influence was $0.5 \mathrm{~V} / \mathrm{m}$ when stimulating at $1.0 \mathrm{~mA}$ using a device similar to the Neuroelectrics StarStim device used in this study (Opitz et al., 2016), and $0.4 \mathrm{~V} / \mathrm{m}$ when stimulating at $2.0 \mathrm{~mA}$ using the NeuroConn DC Stimulator Plus (neuroCare; Huang et al., 2017). This suggests our approach has a better chance of inducing measurable oscillatory effects intracranially compared with previous work.

One other study, to our knowledge, has investigated a version of closed-loop tACS with the intent of enhancing overnight memory consolidation. Lustenberger et al. (2016) applied $12 \mathrm{~Hz}$ tACS triggered by endogenous spindle (11-16 Hz) activity and found a positive influence of stimulation on behavior in a motor memory task but not a declarative memory task. Our study provides a more precise closed-loop system by matching the dominant frequency and ongoing phase within the SW band (0.5-1.2 $\mathrm{Hz}$ ), and validates the influence on behavior in a target detection task that is similar to many declarative memory tasks. Moreover, we find effects for a stimulation-induced refractory period that decreases SW power relative to sham but does not disrupt the influence of stimulation on behavior. These results suggest that the longer the stimulation-induced increases in power persist, the better the postsleep performance on the generalized images relative to the repeated images. Further investigations into this phenomenon could provide insight into individual differences in stimulation-induced power changes and its subsequent relationship with behavior.

\section{Spindle coupling}

Here a marginally significant (clusterwise $p=0.053$ ) increase in coupling between the phase of the SW band and the amplitude of high spindles for the active condition was found, and this increase was similar to the stimulation-induced window of spatial and temporal increases in SW power that correlated with behavioral improvements. This is consistent with recent results of sleepdependent consolidation; however, the preferred phase in our results is $180^{\circ}$ out of phase with the SW up state (Ladenbauer et al., 2017; Helfrich et al., 2018). Further, a correlation with behavior was not found in these coupling measures; however, the overlap in time and spatial topography with the stimulation-induced changes in SW power that correlated with behavior suggest that the increased coupling is potentially related to the behavioral changes. Perhaps the specificity of the high-power spindle events used in the modulation strength metric was too broadly defined to reveal correlations with the behavioral measures of interest and center on the SW up state. Further investigations into these stimulation-induced effects are necessary to integrate with existing results.

\section{Conclusions}

In this work and the study by Jones et al. (2018), we focused on building a closed-loop system to better target endogenous oscillations during sleep in hopes of increasing the efficacy of transcranial stimulation on hypothesized systems-level consolidation mechanisms. Through this intervention, we showed behavioral changes that were consistent with consolidation theories, and poststimulation changes in EEG that suggest stimulation-induced enhancement of SW oscillations that positively correlated with those behavioral changes. An increase in SW to spindle coupling was found in an overlapping temporal and spatial topography to the stimulation-induced increases in power and correlation with behavior, suggesting that enhanced SW oscillations lead to an increase in systems-level consolidation processes. We also witnessed apparent stimulation-induced decreases in SW power and coupling with spindles that suggest a reset or refractory period in the endogenous SW oscillations. The positive correlation with behavior persisted into this refractory period, however, and may suggest that individuals less prone to this decrease ultimately show the most improvement in consolidationrelated memory performance. The mechanisms that would explain this relationship with individual differences are unclear, and more investigations into the influence of closed-loop tACS on neuronal processes is required. 


\section{References}

Akerstedt T, Hume K, Minors D, Waterhouse J (1994) The subjective meaning of good sleep, an intraindividual approach using the Karolinska Sleep Diary. Percept Mot Skills 79:287-296. CrossRef Medline

Bates D, Mächler M, Bolker B, Walker S (2015) Fitting linear mixed-effects models using lme4. J Stat Softw 67:1-48. CrossRef

Berens P (2009) CircStat: a MATLAB toolbox for circular statistics. J Stat Softw 31:1-21. CrossRef

Berry RB, Gamaldo CE, Harding SM, Brooks R, Lloyd RM, Vaughn BV, Marcus CL (2015) AASM scoring manual version 2.2 updates: new chapters for scoring infant sleep staging and home sleep apnea testing. J Clin Sleep Med 11:1253-1254. CrossRef Medline

Braboszcz C, Delorme A (2011) Lost in thoughts: neural markers of low alertness during mind wandering. Neuroimage 54:3040-3047. CrossRef Medline

Ciuparu A, Mureşan RC (2016) Sources of bias in single-trial normalization procedures. Eur J Neurosci 43:861-869. CrossRef Medline

Clark VP, Coffman BA, Mayer AR, Weisend MP, Lane TD, Calhoun VD, Raybourn EM, Garcia CM, Wassermann EM (2012) TDCS guided using fMRI significantly accelerates learning to identify concealed objects. Neuroimage 59:117-128. CrossRef Medline

Coffman BA, Trumbo MC, Clark VP (2012) Enhancement of object detection with transcranial direct current stimulation is associated with increased attention. BMC Neurosci 13:108. CrossRef Medline

Delorme A, Makeig S (2004) EEGLAB: an open source toolbox for analysis of single-trial EEG dynamics including independent component analysis. J Neurosci Methods 134:9-21. CrossRef Medline

Dickinson DL, Cazier J, Cech T (2016) A practical validation study of a commercial accelerometer using good and poor sleepers. Health Psychol Open 3:2055102916679012. CrossRef Medline

Dvorak D, Fenton AA (2014) Toward a proper estimation of phaseamplitude coupling in neural oscillations. J Neurosci Methods 225:42-56. CrossRef Medline

Falcone B, Coffman BA, Clark VP, Parasuraman R (2012) Transcranial direct current stimulation augments perceptual sensitivity and 24-hour retention in a complex threat detection task. PLoS One 7:e34993. CrossRef Medline

Fischer S, Drosopoulos S, Tsen J, Born J (2006) Implicit learning-explicit knowing: a role for sleep in memory system interaction. J Cogn Neurosci 18:311-319. CrossRef Medline

Helfrich RF, Mander BA, Jagust WJ, Knight RT, Walker MP (2018) Old brains come uncoupled in sleep: slow wave-spindle synchrony, brain atrophy, and forgetting. Neuron 97:221-230.e4. CrossRef Medline

Hopstaken JF, van der Linden D, Bakker AB, Kompier MA (2015) A multifaceted investigation of the link between mental fatigue and task disengagement. Psychophysiology 52:305-315. CrossRef Medline

Horvath JC, Forte JD, Carter O (2015) Evidence that transcranial direct current stimulation (tDCS) generates little-to-no reliable neurophysiologic effect beyond MEP amplitude modulation in healthy human subjects: a systematic review. Neuropsychologia 66:213-236. CrossRef Medline

Huang Y, Liu AA, Lafon B, Friedman D, Dayan M, Wang X, Bikson M, Doyle WK, Devinsky O, Parra LC (2017) Measurements and models of electric fields in the in vivo human brain during transcranial electric stimulation. Elife 6:e18834. CrossRef Medline

Jacobson L, Koslowsky M, Lavidor M (2012) tDCS polarity effects in motor and cognitive domains: a meta-analytical review. Exp Brain Res 216:1-10. CrossRef Medline

Jefferys JG, Deans J, Bikson M, Fox J (2003) Effects of weak electric fields on the activity of neurons and neuronal networks. Radiat Prot Dosimetry 106:321-323. CrossRef Medline

Ji D, Wilson MA (2007) Coordinated memory replay in the visual cortex and hippocampus during sleep. Nat Neurosci 10:100-107. CrossRef Medline

Jones AP, Choe J, Bryant NB, Robinson CSH, Skorheim SW, Combs A, Lamphere ML, Robert B, Gill HA, Heinrich MD, Ketz NA, Howard MD, Clark VP, Pilly PK (2018) Effects of closed-loop tACS during slow-wave sleep oscillations enhances consolidation of generalized information. Manuscript in preparation.
Kuznetsova A, Brockhoff PB, Christensen RHB (2015) Package 'lmerTest'. Vienna, Austria: R Foundation for Statistical Computing.

Ladenbauer J, Ladenbauer J, Külzow N, de Boor R, Avramova E, Grittner U, Flöel A (2017) Promoting sleep oscillations and their functional coupling by transcranial stimulation enhances memory consolidation in mild cognitive impairment. J Neurosci 37:7111-7124. CrossRef Medline

Lafon B, Henin S, Huang Y, Friedman D, Melloni L, Thesen T, Doyle W, Buzsáki G, Devinsky O, Parra LC, A Liu A (2017) Low frequency transcranial electrical stimulation does not entrain sleep rhythms measured by human intracranial recordings. Nat Commun 8:1199. CrossRef Medline

Lustenberger C, Boyle MR, Alagapan S, Mellin JM, Vaughn BV, Fröhlich F (2016) Feedback-controlled transcranial alternating current stimulation reveals a functional role of sleep spindles in motor memory consolidation. Curr Biol 26:2127-2136. CrossRef Medline

Maris E, Oostenveld R (2007) Nonparametric statistical testing of EEG- and MEG-data. J Neurosci Methods 164:177-190. CrossRef Medline

Marshall L, Mölle M, Hallschmid M, Born J (2004) Transcranial direct current stimulation during sleep improves declarative memory. J Neurosci 24:9985-9992. CrossRef Medline

Marshall L, Helgadóttir H, Mölle M, Born J (2006) Boosting slow oscillations during sleep potentiates memory. Nature 444:610-613. CrossRef Medline

Matzen LE, Benz ZO, Dixon KR, Posey J, Kroger JK, Speed AE (2010) Recreating Raven's: software for systematically generating large numbers of raven-like matrix problems with normed properties. Behavior Res Methods 42:525-541. CrossRef

Ngo HV, Miedema A, Faude I, Martinetz T, Mölle M, Born J (2015) Driving sleep slow oscillations by auditory closed-loop stimulation-a selflimiting process. J Neurosci 35:6630-6638. CrossRef Medline

Oldfield RC (1971) The assessment and analysis of handedness: Edinburgh Handedness Inventory. Neuropsychologia 9:97-113. CrossRef Medline

Oostenveld R, Fries P, Maris E, Schoffelen JM (2011) FieldTrip: open source software for advanced analysis of MEG, EEG, and invasive electrophysiological data. Comput Intell Neurosci 2011:156869. CrossRef Medline

Opitz A, Falchier A, Yan CG, Yeagle EM, Linn GS, Megevand P, Thielscher A, Deborah AR, Milham MP, Mehta AD, Schroeder CE, et al (2016) Spatiotemporal structure of intracranial electric fields induced by transcranial electric stimulation in humans and nonhuman primates. Sci Rep 6:31236. CrossRef Medline

Prehn-Kristensen A, Munz M, Göder R, Wilhelm I, Korr K, Vahl W, Wiesner CD, Baving L (2014) Transcranial oscillatory direct current stimulation during sleep improves declarative memory consolidation in children with attention-deficit/hyperactivity disorder to a level comparable to healthy controls. Brain Stimul 7:793-799. CrossRef Medline

Rasch B, Born J (2013) About sleep's role in memory. Physiol Rev 93:681766. CrossRef Medline

R-Core-Team (2015) R: A language and environment for statistical computing [Internet]. Vienna, Austria: R Foundation for Statistical Computing.

Reato D, Rahman A, Bikson M, Parra LC (2010) Low-intensity electrical stimulation affects network dynamics by modulating population rate and spike timing. J Neurosci 30:15067-15079. CrossRef Medline

Ruhnau P, Rufener KS, Heinze HJ, Zaehle T (2018) Sailing in a sea of disbelief: in vivo measurements of transcranial electric stimulation in human subcortical structures. Brain Stimul 11:241-243. CrossRef Medline

Staresina BP, Bergmann TO, Bonnefond M, van der Meij R, Jensen O, Deuker L, Elger CE, Axmacher N, Fell J (2015) Hierarchical nesting of slow oscillations spindles and ripples in the human hippocampus during sleep. Nat Neurosci 18:1679-1686. CrossRef Medline

Stickgold R, Walker MP (2013) Sleep-dependent memory triage: evolving generalization through selective processing. Nat Neurosci 16:139-145. CrossRef Medline

Tamminen J, Payne JD, Stickgold R, Wamsley EJ, Gaskell MG (2010) Sleep spindle activity is associated with the integration of new memories and existing knowledge. J Neurosci 30:14356-14360. CrossRef Medline

Wagner U, Gais S, Haider H, Verleger R, Born J (2004) Sleep inspires insight. Nature 427:352-355. CrossRef Medline

Westerberg CE, Florczak SM, Weintraub S, Mesulam MM, Marshall L, Zee PC, Paller KA (2015) Memory improvement via slow-oscillatory stimulation during sleep in older adults. Neurobiol Aging 36:2577-2586. CrossRef Medline 